\title{
Pharmacist Prescribing for Minor Ailments Service Development: The Experience in Ontario
}

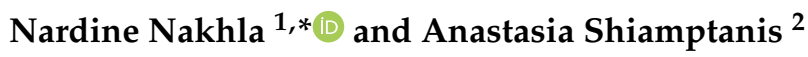 \\ 1 School of Pharmacy, University of Waterloo, 10A Victoria St. S., Kitchener, ON N2G 1C5, Canada \\ 2 Ontario College of Pharmacists, 483 Huron St., Toronto, ON M5R 2R4, Canada; \\ anastasiapharmd@hotmail.com \\ * Correspondence: nardine.nakhla@uwaterloo.ca; Tel.: +1-519-888-4567 (ext. 21340)
}

Citation: Nakhla, N.; Shiamptanis, A. Pharmacist Prescribing for Minor Ailments Service Development: The Experience in Ontario. Pharmacy 2021, 9, 96. https://doi.org/10.3390/ pharmacy 9020096

Academic Editor: Hamde Nazar

Received: 30 March 2021

Accepted: 22 April 2021

Published: 27 April 2021

Publisher's Note: MDPI stays neutral with regard to jurisdictional claims in published maps and institutional affiliations.

Copyright: (c) 2021 by the authors. Licensee MDPI, Basel, Switzerland. This article is an open access article distributed under the terms and conditions of the Creative Commons Attribution (CC BY) license (https:// creativecommons.org/licenses/by/ $4.0 /)$.

\begin{abstract}
To date, eight of ten Canadian provinces have authorized pharmacists to prescribe for minor ailments. Prompted by a request by the Ontario Minister of Health, draft regulations were submitted to enable this pharmacy service in Ontario. Differences exist in how jurisdictions have approached development and delivery of these programs. This paper will summarize key differences and similarities among existing programs while highlighting the multi-pronged approach utilized by Ontario. Such an approach involved broad stakeholder engagement, implementation science, and an evaluations framework to guide an assessment of the impact of this new service. These insights can be leveraged by other jurisdictions planning to initiate or evolve their minor ailment prescribing services.
\end{abstract}

Keywords: minor ailments; minor illness; self-limiting conditions; low acuity conditions; self-care; ambulatory conditions; pharmacist prescribing; scope of practice; advanced pharmacy practice; access to care

\section{Introduction}

The current COVID-19 pandemic has highlighted the unprecedented need to consider more multifaceted, sustainable solutions for healthcare globally. Pharmacists are Canada's third largest group of healthcare professionals (HCPs), with approximately 45,651 licensed pharmacists across the nation [1]. Pharmacists possess extensive knowledge and skills in pharmacotherapeutics that can be optimized to narrow disparities and facilitate access to high-quality healthcare. For example, pharmacists have a long history of helping patients manage minor ailments. Traditionally, this has involved making non-prescription medication recommendations, facilitating other self-care decisions (e.g., lifestyle changes), and referring patients to other HCPs when needed. As one of the most accessible HCPs, pharmacists are well-positioned to strengthen community-based care for a variety of conditions. Canadian estimates show that 10 to $30 \%$ of physician consultations are for minor ailments [2]; United Kingdom (UK) estimates are higher, suggesting that between $18 \%$ and $37 \%$ of general practitioner consultations are for minor ailments, many of which can be managed by pharmacists [3].

In recent years, several countries introduced "pharmacist prescribing for minor ailments" (PPMA) in an effort to increase access to primary healthcare and reduce the burden of minor ailment care on other settings (e.g., emergency departments (EDs), urgent care) by leveraging the clinical capabilities of pharmacists and providing patients the choice of receiving care from community pharmacists [4,5]. PPMA programs promote efficiencies within the healthcare system by enabling pharmacists to prescribe or recommend the most appropriate treatment for certain minor ailments, reducing the need to refer to another HCP and setting for a prescription [6]. This affords patients with the opportunity to receive timely care in the community from highly accessible and trained HCPs. A systematic review by Paudyal et al. concluded that the low reconsultation and high symptom-resolution 
rates of up to $94 \%$ associated with PPMA programs suggest that these services are being appropriately managed by pharmacists and do not compromise patient safety or quality of care [4]. Furthermore, a 2014 review supported community pharmacy-based minor ailment services as an effective and efficient strategy for managing patients that may reduce strain on healthcare systems and thus facilitate sustainability $[7,8]$.

PPMA programs exist in some Canadian provinces and in some parts of the UK (i.e., Wales, England, Scotland, and Northern Ireland) [2,9-15]. Their feasibility is currently being considered in Spain, New Zealand, and Australia [16]. The term "pharmacist prescribing for minor ailments" (PPMA) is commonly used across Canada to depict the community pharmacy service that entails assessment and prescribing of medications to manage minor ailments. However, the term "minor ailment service" (MAS) and "common ailment schemes" (CAS) are used to characterize similar services globally. For this paper, the term PPMA is used interchangeably for all regions and countries.

PPMA services differ in their structural design and service delivery regulations from country to country and even from jurisdictions within the same country [2,6]. Aly et al. recently performed the first international comparative review of minor ailment service features and outlined essential elements to consider when designing such a program [6]. At the time of publication, Ontario submitted draft regulations to the government to enable this type of pharmacy service. The aim of this paper is to describe the process utilized by Ontario, which incorporates data analysis, broad stakeholder engagement, patient involvement, implementation science, and an evaluations framework. Through this experience, we build on the work done by Aly et al. and propose additional factors to consider when developing and implementing PPMA programs [6].

\section{Ontario: Constructing the Approach}

In May 2019, as part of an overall approach to expand the scope of HCPs, the Minister of Health directed the Ontario College of Pharmacists (the College) to submit draft regulations by June 2020 to enable pharmacists to prescribe drugs for certain minor ailments to improve access to routine care for patients in the community and reduce the need for emergency or urgent care visits [17]. This request occurred after a 2019 report commissioned by the government, Improving Healthcare and Ending Hallway Medicine, that provided recommendations on creating a more efficient healthcare system in which patients can access care where and when it is needed [18]. Soon thereafter, and consistent with the College's mission of driving quality and safe pharmacy care and improved outcomes, the College developed a multi-pronged system- and outcomes-focused approach to drafting the regulations. This included working closely with the Ministry of Health throughout the process to align policy direction and key regulatory changes. In positioning the PPMA program to meet the Ministry's objective of providing more pathways for patients to access care in the community, the College felt it was important to consider several factors, including experiences from other jurisdictions, the perspectives of those most impacted by these changes (i.e., patients, pharmacists, physicians), and the healthcare system as a whole.

The key pillars of the multi-pronged approach will be described below and included defining the regulatory framework, establishing an expert group, and patient involvement and broad engagement.

\subsection{Defining the Regulatory Framework}

In Ontario, regulations to enable pharmacists prescribing for smoking cessation were added to the Pharmacy Act, 1991 in 2012. As such, a framework on general expectations for prescribing was devised at that time. This included notifying the primary care provider (PCP) after prescribing, providing the patient with the option of dispensing the medication at another pharmacy, and assessing documentation expectations. The amendments are therefore built on the existing regulations related to prescribing for smoking cessation. The provisions were reviewed to determine if additional prescribing expectations specific to minor ailments were needed. Additionally, it was important to consider if there are any 
existing regulatory provisions that may impact the policy direction. For example, in Ontario, the College has the authority to enable pharmacists to prescribe specific medications or medication categories, whereas other provinces allow for broader prescribing.

In a jurisdiction lacking the foundational framework for policy development, we suggest drawing from the profession's standards of practice, code of ethics, and any applicable practice directives (e.g., criteria for prescribing, dispensing) issued by the regulatory authority to develop the basis of such a service. Stakeholder engagement is also key in identifying any safeguards needed and refining practice expectations if necessary.

\subsection{Establishing an Expert Advisory Group}

To facilitate expedient and systems-focused discussions, the College assembled a Minor Ailments Advisory Group (MAAG) composed of patient advocates, community pharmacists, physicians, academics, and health systems research experts soon after the Minister's request was made. The expert advisory group provided advice and input to the College on key regulatory considerations and safety parameters to align the program with the government's objectives while maintaining quality patient care. This involved working on various aspects of planning and development, including draft regulations, proposed minor ailments and associated medication categories, safeguards for implementation, an evaluations framework for prescribing, and practice support tools [19].

The multi-stakeholder composition of MAAG facilitated robust discussions. The group held regular meetings over the course of several months (June 2019 to November 2020) and followed a structured, modified-Delphi approach in providing their advice and guidance to the College on which minor ailments and medication categories to include. Key inputs in the decision-making process included the results of an environmental scan, experience in other provinces, ED administrative data on conditions that potentially could be managed by a pharmacist, and feedback from pharmacy professionals and patients obtained through various mechanisms such as surveys and focus groups. Criteria were agreed upon by MAAG to apply a consistent approach in evaluating which minor ailments to recommend for the minor ailment program [19]. The key actions taken by MAAG are summarized below.

\section{Environmental Scan and Assessment}

MAAG contributed to an environmental scan, which included review of quantitative and qualitative data to explore the current landscape of pharmacist prescribing for minor ailments across various Canadian jurisdictions. Additional data were also collected from the websites of the provincial regulatory authorities as well as provincial and national pharmacy advocacy groups. Through this review, the group considered all of the minor ailments included in any provincial program and studies relating to evaluation of these services. It was noted that terminology of the service and criteria for inclusion varies across jurisdictions, and that there was no universally accepted definition for minor ailments. Table 1 provides a breakdown of differences in prescriptive authority and terminology used across Canada. 
Table 1. Conditions authorized for pharmacist prescribing under minor ailment programs in Canada.

\begin{tabular}{|c|c|c|c|c|c|c|c|c|c|}
\hline Conditions $₫$ & Alberta * & Manitoba & New Brunswick & $\begin{array}{c}\text { Newfoundland } \\
\text { and Labrador }\end{array}$ & Nova Scotia & $\begin{array}{l}\text { Prince Edward } \\
\text { Island }\end{array}$ & Quebec & Saskatchewan & Ontario ** \\
\hline Acne vulgaris $^{a}$ & $X$ & $x$ & $X$ & $\mathrm{X}$ & $X$ & $\mathrm{X}$ & $X$ & $x$ & \\
\hline Acute mountain sickness prevention & $x$ & & & & & & $x$ & & \\
\hline Allergic conjunctivitis & $X$ & & & & & & $\mathrm{X}$ & $x$ & $x$ \\
\hline Atopic dermatitis (eczema) ${ }^{b}$ & $X$ & $x$ & $X$ & $X$ & $X$ & $X$ & $X$ & $X$ & $x$ \\
\hline Bacterial conjunctivitis & $X$ & & & & & & & $X$ & $X$ \\
\hline Calluses and corns & $X$ & $x^{1}$ & $X$ & $X$ & $x$ & $X$ & & & \\
\hline Contraception (hormonal) & $x$ & $x^{1}$ & & & $x^{3}$ & & $X$ & $x$ & \\
\hline Contact allergic dermatitis ${ }^{\mathrm{c}}$ & $X$ & $X$ & $x$ & $X$ & $x$ & $X$ & $X$ & & $x$ \\
\hline Cough & $x$ & & $x$ & $X$ & $x$ & $X$ & & & \\
\hline Candidal stomatitis (oral thrush) ${ }^{d}$ & $X$ & $x$ & $X$ & $X$ & $X$ & $X$ & $x$ & $x$ & $X$ \\
\hline Dandruff & $x$ & & $x$ & $x$ & $x$ & $x$ & & & \\
\hline $\begin{array}{c}\text { Diaper rash/dermatitis, irritant and } \\
\text { candidal }\end{array}$ & $x$ & & & & & & $X$ & $x$ & \\
\hline Diarrhea (noninfectious) & $x$ & & $x$ & $X$ & $X$ & $X$ & & & \\
\hline Dysmenorrhea $\mathrm{e}^{\mathrm{u}}$ & $X$ & $x^{1}$ & $X$ & $X$ & $X$ & $X$ & $\mathrm{X}$ & $x$ & $x$ \\
\hline Dyspepsia (indigestion) & $X$ & & $X$ & $X$ & $X$ & $X$ & $X$ & & \\
\hline Emergency contraception & $x$ & $x^{1}$ & $x$ & $X$ & $X$ & $x$ & $X$ & $x$ & \\
\hline Erectile dysfunction & $x$ & & & & & & & $x$ & \\
\hline Folliculitis & $x$ & & & & & & & $x$ & \\
\hline Gastroesophageal reflux disease & $x$ & $x^{1}$ & $x$ & $x$ & $x$ & $x$ & $x$ & $x$ & $x$ \\
\hline $\begin{array}{l}\text { (neartburn) } \\
\text { Head lice }\end{array}$ & $x$ & & & & & & $x$ & & \\
\hline Headache $\mathrm{g}$ & $X$ & & $x$ & $X$ & $x$ & $\mathrm{X}$ & & $x$ & \\
\hline Hemorrhoids $\mathrm{h}$ & $x$ & $X$ & $x$ & $x$ & $X$ & $X$ & $X$ & $x$ & $X$ \\
\hline Herpes labialis/simplex (cold sores) & $x$ & $x^{1}$ & $x$ & $X$ & $X$ & $X$ & $x$ & $x$ & $x$ \\
\hline Herpes zoster (shingles) prevention & $x$ & $x^{1}$ & & $x^{2}$ & $x^{4}$ & $x^{5}$ & $x$ & $x$ & \\
\hline Impetigo & $x$ & $x^{1}$ & $x$ & $x$ & $x$ & & & $x$ & $X$ \\
\hline Influenza treatment & $X$ & & & & & & $X$ & $X$ & \\
\hline Insect bites ${ }^{\mathrm{i}}$ & $X$ & & $X$ & $X$ & $x$ & $x$ & & $X$ & $x$ \\
\hline Irritant contact dermatitis ${ }^{c}$ & $X$ & $x$ & & $X$ & & & & & $X$ \\
\hline Joint pain (minor or mild) ${ }^{j}$ & $x$ & & $X$ & $X$ & $X$ & $X$ & & & \\
\hline Malaria prevention & $x$ & & & & $x^{3}$ & & $x$ & & \\
\hline Mouth/oral/aphthous ulcers ${ }^{k}$ & $x$ & $x$ & $x$ & $x$ & $x$ & $x$ & $x$ & $x$ & \\
\hline Muscle pain (minor or mild) ${ }^{j}$ & $x$ & & $x$ & $x$ & $X$ & $X$ & & $x$ & $X$ \\
\hline Nasal congestion $\mathrm{p}$ & $x$ & & $x$ & $X$ & $x$ & $x$ & & & \\
\hline Nausea & $x$ & & $x$ & & $x$ & $X$ & $x$ & & \\
\hline
\end{tabular}


Table 1. Cont.

\begin{tabular}{|c|c|c|c|c|c|c|c|c|c|}
\hline Conditions $₫$ & Alberta * & Manitoba & New Brunswick & $\begin{array}{c}\text { Newfoundland } \\
\text { and Labrador }\end{array}$ & Nova Scotia & $\begin{array}{c}\text { Prince Edward } \\
\text { Island }\end{array}$ & Quebec & Saskatchewan & Ontario ** \\
\hline Nausea and Vomiting & $\mathrm{X}$ & & & $\mathrm{X}$ & & & $\mathrm{X}$ & & \\
\hline Nausea/vomiting of pregnancy ${ }^{1}$ & $X$ & $X$ & & & & & $X$ & & \\
\hline Obesity & $X$ & & & & & & & $X$ & \\
\hline Onychomycosis & $\mathrm{X}$ & & & & & & & $x$ & \\
\hline Pinworms/threadworms & $X$ & $x^{1}$ & $X$ & $X$ & $X$ & $X$ & $X$ & & \\
\hline $\begin{array}{c}\text { Pregnancy (requiring prenatal } \\
\text { vitamins) }\end{array}$ & $x$ & & & & & & $x$ & & \\
\hline Seborrhoeic dermatitis $\mathrm{m}$ & $X$ & $X$ & & $\mathrm{X}$ & & & & & \\
\hline $\begin{array}{l}\text { Smoking cessation/nicotine } \\
\text { dependence }\end{array}$ & $X$ & $X$ & $x$ & $x$ & $x$ & $x$ & $\mathrm{X}$ & $X$ & $X$ \\
\hline Sore throat $\mathrm{p}$ & $x$ & & $x$ & $x$ & $x$ & $x$ & $x$ & & \\
\hline Tinea corporis infection (ringworm) ${ }^{\mathrm{f}}$ & $X$ & $X^{1}$ & $X$ & $x$ & $x$ & $X$ & $x$ & $X$ & \\
\hline Tinea cruris infection (jock itch) ${ }^{f}$ & $X$ & $X^{1}$ & $X$ & $X$ & $X$ & $X$ & $X$ & $X$ & \\
\hline Tinea pedis infection (athlete's foot) ${ }^{\mathrm{f}}$ & $X$ & $X$ & $X$ & $X$ & $X$ & $X$ & & $X$ & \\
\hline Upper respiratory tract conditions $q$ & $X$ & & $X$ & $X$ & & & & & \\
\hline Urinary tract infection (UTIs) ${ }^{\mathrm{r}}$ & $x$ & $x^{1}$ & $x$ & & $x^{4}$ & $X$ & $X$ & $X$ & $X$ \\
\hline Urticaria $^{\mathrm{i}}$ & $X$ & $\mathrm{X}$ & $X$ & $X$ & $\mathrm{X}$ & $X$ & $X$ & & $\mathrm{X}$ \\
\hline Vaginal candidiasis (yeast infection) & $x$ & $x^{1}$ & $x$ & $x$ & $x$ & $x$ & $x$ & & \\
\hline Viral conjunctivitis & $X$ & & & & & & & & $X$ \\
\hline Warts ${ }^{\mathrm{s}}$ & $X$ & $x^{1}$ & $X$ & $X$ & $X$ & $X$ & & & \\
\hline Xerophthalmia (dry eyes) & $X$ & & $X$ & $X$ & $X$ & $X$ & & & \\
\hline
\end{tabular}

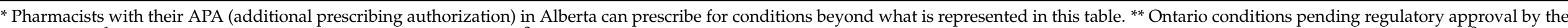

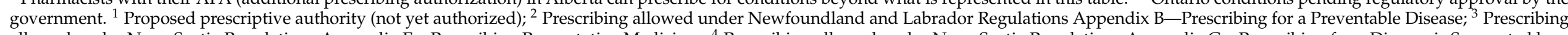

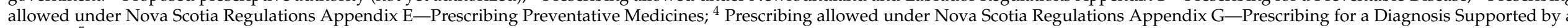

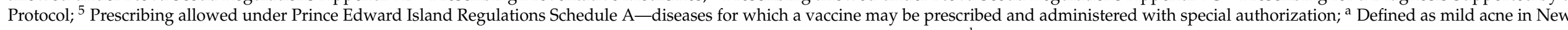

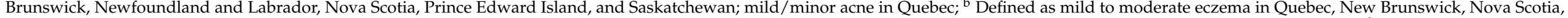

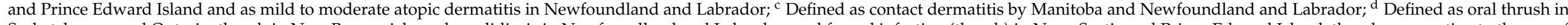

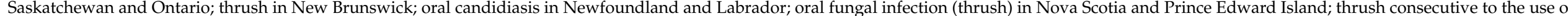

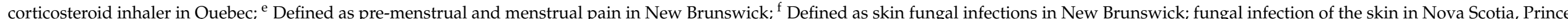

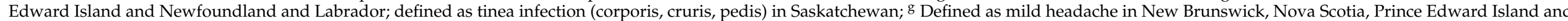

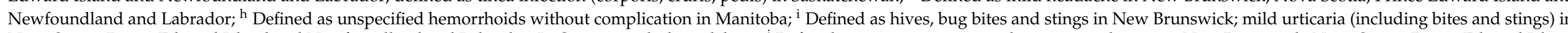

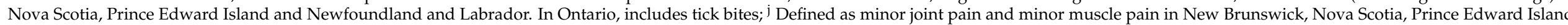

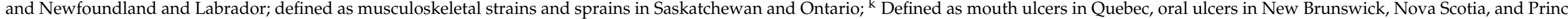

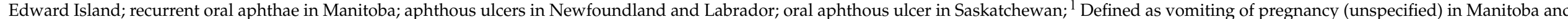

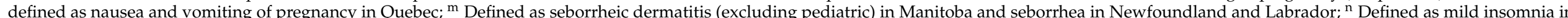

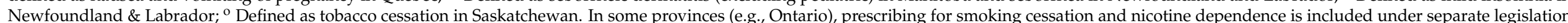

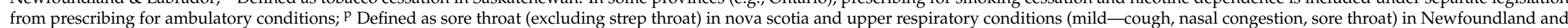




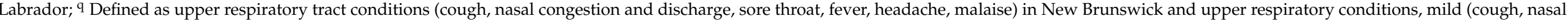

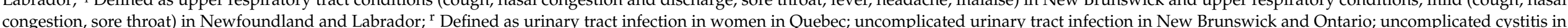

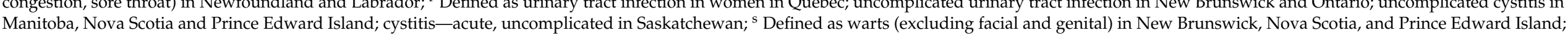

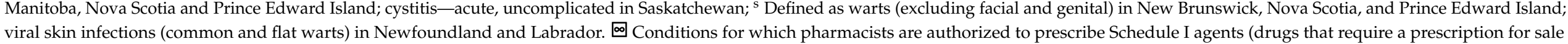
pursuant to an assessment by a healthcare provider who is authorized to prescribe). 
It was the consensus of the MAAG to align the term "minor ailment" with the definition used in Nova Scotia and Newfoundland and Labrador, as well as those found in the literature [16,20-22]:

Minor ailments are health conditions that can be managed with minimal treatment and/or self-care strategies. Additional criteria include:

- usually a short-term condition;

- $\quad$ lab tests are not usually required;

- low risk of treatment masking underlying conditions;

- medication and medical histories can reliably differentiate more serious conditions;

- $\quad$ only minimal or short-term follow-up is required.

Definitions which included the term "self-limited" (e.g., Saskatchewan, Manitoba) were not adopted in their entirety as some conditions, such as urinary tract infections, may not fully resolve without treatment. Any condition that did not meet this definition were not considered by MAAG for inclusion under these regulations.

The studies identified from the environmental scan were used to draw upon the experience of other Canadian provinces in implementing PPMA programs. MAAG applied learnings from the experiences in other jurisdictions in making recommendation for the program. For example, the main reported barriers to adoption of PPMA services were integration into workflow, patient awareness, lack of sufficient revenue, and lack of time $[23,24]$. In alignment with the Institute of Healthcare Improvement Quadruple Aim, MAAG reviewed studies reporting on the impact on patient experience and health system efficiency $[25,26]$. Patients avoided physician and ED visits as the result of provincial PPMA programs [27] and were able to access care sooner [28]. Patients reported that they are satisfied with the service $[27,29,30]$.

Key findings from the environmental scan were applied to the Capabilities, Opportunities, Motivation, and Behavior model (COM-B) to assist MAAG with identifying and recommending strategies for change, including regulation, policy, implementation, and evaluation. Refer to Section 3.1.3 for additional information.

Additionally, information on conditions that are resulting in ED visits that can be safely managed through pharmacist prescribing was also reviewed to help ground the policy in current and emerging healthcare needs and maximize the benefits to patients and the broader health system [31].

After building this evidence-based foundation, MAAG used a conceptual framework (Appendix A Figure A1) with criteria to guide decision-making for selecting which minor ailments to recommend for inclusion in the PPMA program. The criteria included: likelihood of preventing urgent care visits; treatment commonly involves the option of a prescription medication; time-sensitive conditions; and those frequently included in other provinces. The MAAG followed a three-round modified-Delphi approach in reviewing the minor ailments. First, each MAAG member utilized the criteria and prioritized the conditions based on high, moderate, and low priority for implementation, and those that do not meet the definition. The collated results were shared with the group, and a second round of prioritization occurred via an anonymous web-based questionnaire. After this second round, conditions with variation were discussed by the group and final agreement was then obtained at an in-person consensus meeting (round three). Through this process, MAAG refined the full list of conditions approved in at least one province to a list of 18 , which were circulated for broad feedback.

A similar three-round modified-Delphi approach was taken to provide recommendations for medication categories. The MAAG reviewed the literature and guidelines to identify the most common treatment options for the minor ailments and considered both the risk level of the medications and the level of follow-up required in making a final recommendation to the College. 


\subsection{Patient Involvement}

A cornerstone to the approach was the involvement of patients throughout the process. In addition to patient involvement on the MAAG, the College conducted third-party facilitated focus groups to obtain feedback on the list of minor ailments and other safety considerations. Through these focus groups, key implementation considerations were highlighted such as the importance of communication between HCPs to support continuity of care and the need for the practice environment to be conducive to the provision of the PPMA service. Additional public input was sought through the Citizen Advisory Group (CAG), a collaborative partnership that brings together patients and caregivers to provide feedback related to health regulation in Ontario [32]. The CAG provided insight on which minor ailments they would be most likely to see a pharmacist for, which included urinary tract infections, hemorrhoids, conjunctivitis, and insect bites [32]. Meaningful patient involvement was key to the regulatory development process as it directed the program to areas most important to patients.

\subsection{Broad Stakeholder Engagement}

The request to expand the scope of practice of pharmacists recognizes the role of the profession in collaborating to improve Ontario's health system outcomes. It is therefore important to understand, from the perspective of both the public and other professions, where prescriptive authority for minor ailments for pharmacists will have the greatest impact to improve system and patient outcomes. To achieve this understanding, the College engaged extensively throughout the regulatory development process leading up to, and including, the final open consultation on the proposed amendments to provincial regulations.

The objectives of the PPMA service were shared at each point to enable stakeholders to contribute insights to help achieve the overall system goals. A survey was administered to pharmacy professionals early in the process to inform the policy direction. Pharmacy professionals and students were asked through the survey to provide feedback on the preliminary list of 18 conditions. Furthermore, the College engaged in discussions with health professional associations, including pharmacy, medicine, and nursing, and collaborated with other health regulatory colleges.

The College also obtained feedback from stakeholders through the open consultation process, which ensures that the public, practitioners, and all other interested stakeholders have an opportunity to provide feedback on new and devised documents related to pharmacy practice prior to final approval. Feedback was received from pharmacy professionals, pharmacy students, the public, associations representing pharmacists, physicians, and family health teams, as well as pharmacy organizations. Together, these perspectives provided important insights that helped to inform regulatory drafting and the implementation strategy. Further refinement to the list of medication categories also occurred based on feedback obtained [33].

\section{Ontario: The Final Results}

\subsection{PPMA Service Components}

Triangulation of the results of the environmental scan along with health administrative data and broad engagement coalesced to produce results that informed the structural design. A description of the structural design features and delivery of the PPMA service can be found below.

\subsubsection{List of Minor Ailments and Medication Categories}

Based on the feedback received, and to align with the Ministry's direction, the preliminary list of 18 ailments was further refined to 12:

- Urinary tract infection (uncomplicated)

- Dermatitis (atopic/eczema, allergic and contact skin rashes)

- Insect bites (including tick bites) and urticaria (hives) 
- Conjunctivitis (bacterial, allergic, viral)

- Allergic rhinitis (nasal symptoms from allergies)

- Candidal stomatitis (oral thrush)

- Herpes labialis (cold sores)

- Hemorrhoids

- Gastroesophageal reflux disease (GERD)

- Dysmenorrhea (menstrual cramps)

- Musculoskeletal sprains and strains

- Impetigo (bacterial skin infection common in children)

Some of the conditions initially proposed were not eliminated entirely but rather grouped together based on therapeutic similarities. For example, prescribing for postexposure prophylaxis of Lyme disease from tick bites was moved to the insect bites category. Tick bites was added to the list due to the importance of timely access to prophylaxis treatment and the high-risk areas for Lyme disease in the province, which include remote locations [34].

The agents authorized for pharmacist prescribing to manage the 12 ailments, if treatment is considered necessary and appropriate, are outlined in the draft regulations according to the American Hospital Formulary Service (AHFS) classification system. Considerations such as recent evidence, clinical practice guidelines, best practices, and antimicrobial stewardship guided the final selection of the medication categories.

\subsubsection{Safeguards}

Elements to support safe implementation identified through the consultations included collaboration, patient assessment, and consideration of any conflict-of-interest involvement in prescribing. The safeguards in place (i.e., current regulations for prescribing, standards of practice, code of ethics) in Ontario address these factors.

Two additional elements were added to the regulation to address common feedback received around ensuring that pharmacists are aware of the practice expectations, such as referral to another HCP when appropriate. To assist pharmacists with understanding, interpreting, and applying the regulations to their practice, the College Board approved a mandatory orientation module. This short online module reinforces important practice expectations, such as referring to an appropriate HCP for patients presenting with "red flags", and highlights the expected purposes of the service. PPMA will become a general practice expectation of pharmacists in Ontario. As such, all practicing pharmacists are required to complete this module within one year of its availability. Furthermore, previous work in this area noted that to assist with sustainability of a PPMA program, it is important for the profession to be clear on and understand the objectives of the service [35,36]. Clinical education is not mandated and, as with any practice development, pharmacists are responsible for self-assessing their need for additional continuing education to ensure that they have the required knowledge, skill, and judgement required to provide quality patient care. Both pharmacy university programs in Ontario-and all ten across Canada-include minor ailments in their curricula [37]. Additionally, Ontario pharmacies are required to have the references and resources needed for pharmacists to apply best practices and evidence-based care. Information on the assessment and treatment for minor ailments as well as algorithms are available in Canadian drug references. The orientation module directs pharmacists to these resources and reinforces expectations for the use of evidence and guidelines. The use of guidelines and algorithms to support clinical decision making were identified as important facilitators for supporting quality patient care [35].

Moreover, to support pharmacists in their role as antimicrobial stewards, the MAAG recommended that resources (i.e., concise practice tools) be developed to facilitate assessment and appropriate antibiotic prescribing for uncomplicated UTIs and Lyme disease post-exposure prophylaxis from tick bites in Ontario. These resources are currently under development by a MAAG subgroup. 


\subsubsection{Implementation and Evaluation}

Key findings from the environmental scan were applied to an implementation framework (the behavioral change wheel) to assist MAAG with identifying and recommending implementation strategies.

Following a framework provides a theory-informed structure to optimize the uptake of interventions. This is done by tailoring implementation strategies to the barriers and facilitators to the behavior change. The COM-B model is central to the behavior change wheel framework. COM-B helped to elucidate the barriers and facilitators according to three determinants of behavior change, capability, opportunity, and motivation, and the subsequent identification and alignment of levers for adoption for PPMA services. Using a model enabled a more in-depth understanding of what may influence the behavior change, in this case, engaging in prescribing for minor ailments, in order to determine implementation strategies that will address those influencers or barriers and facilitators. For example, concerns related to work-load pressures and the ability to meet practice expectations are potential barriers than can relate to the opportunity domain of COM-B. According to the behavior change wheel, effective strategies should involve communication and address environmental concerns. The implementation strategy for PPMA therefore includes a robust communications plan to inform the profession and public of this service, as well as a plan to address concerns with the practice environment that were previously identified through other stakeholder engagement activities. The College initiated a community practice initiative to explore and better understand the workplace challenges and further strengthen the quality and safety of pharmacy care in the province.

Along with an implementation plan, an evaluations framework was also developed (see Figure 1) by MAAG. The framework, depicted in a Logic Model, is intended to guide the development of a plan to measure the impact of the PPMA program on system and patient outcomes, which may involve studying symptom resolution and reconsultation rates. The results from these evaluations will help elucidate the impact on patient safety, which is critical to the determination of further scope enhancements. Additionally, consistent with continuous quality improvement principles, the evaluation may help identify opportunities to optimize how the program is administered.

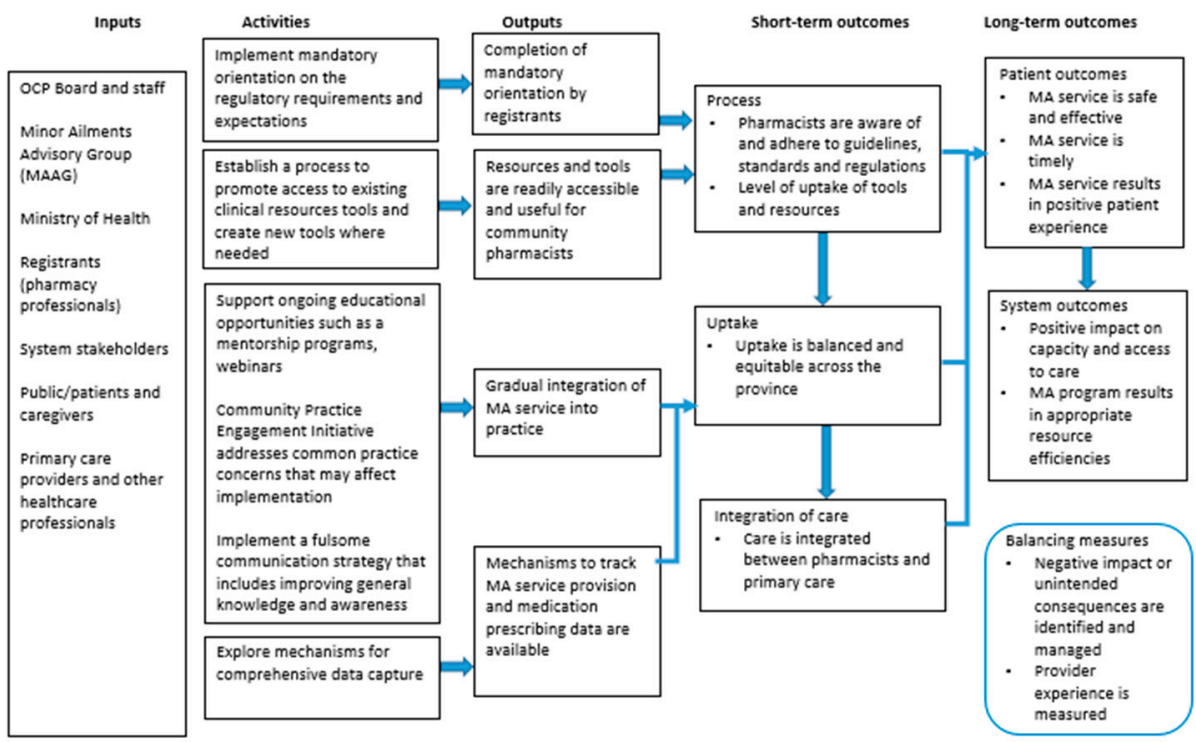

Figure 1. Logic Model for Pharmacist Prescribing for Minor Ailments (PPMA).

\section{Discussion}

Enabling pharmacists to prescribe for minor ailments will allow them to take on a greater role within the healthcare system, to provide patients with streamlined care pathways, and to improve access to minor ailment care in the community. 
While the potential benefits of PPMA services are clearly documented in the literature, there are much less surrounding optimal structures and strategies for building an evidencebased model that enables the reported positive service outcomes. PPMA services differ in their service delivery regulations and structural design from country to country and even from jurisdictions within the same country [2,6]. In this section, we will review similarities and differences between our Ontario model and PPMA services that exist in other jurisdictions.

In the UK, PPMA services authorize pharmacists to supply medications through locally agreed protocols or by supplementary and independent prescribing [3]. In Canada, Saskatchewan pharmacists must prescribe according to protocols that are established for each minor ailment. In Manitoba, pharmacists can prescribe medications in specific medication categories for specific minor ailments without a requirement to follow a standard prescribing protocol. Other provinces (e.g., New Brunswick, Prince Edward Island, Nova Scotia, Newfoundland and Labrador) allow broad prescriptive authority for the minor ailments whereby pharmacists can prescribe any medication that is indicated for the minor ailment. Alberta follows a different model and does not follow a formal PPMA program. Pharmacists may obtain an additional prescribing authority (APA) designation that permits the initiation of any Schedule 1 therapy (with the exception of narcotics and other controlled substances) for any condition. As such, Alberta does not confine pharmacist prescribing to medication categories, medical conditions, or protocols. Furthermore, there are some variations in nomenclature and classification of minor ailments. For example, Manitoba uses the term "self-limiting conditions" while Saskatchewan uses "minor ailments and patient self-care" to describe PPMA services. Differences also exist in the nomenclature of the minor ailments and in classifying which conditions are included under the PPMA service umbrella. For example, canker sores are referred to as "oral ulcers" in Prince Edward Island, "recurrent oral aphthae" in Manitoba, and "aphthous ulcers" in Newfoundland and Labrador. In some provinces (e.g., New Brunswick, Saskatchewan), urinary tract infection is included under the PPMA service, whereas in Nova Scotia, pharmacists can prescribe for the condition; however, this is outside of the minor ailment specific regulation. Currently, only Alberta, Saskatchewan, and Quebec publicly fund the service. There is therefore considerable variation nationally in how the PPMA services are designed, administered, regulated, and funded [2].

Similar to Aly et al., engagement with a variety of key stakeholder groups was an integral component weaved throughout the service design process [6]. This broad stakeholder engagement offered insight related to various perspectives of the key stakeholders, illuminated the potential impacts on different areas of the healthcare system, and ultimately informed the policy direction. As was found in the study exploring the perceptions of stakeholders on a national common ailment service in Wales, engaging stakeholders in the early stages of the pharmacy-based program increased sense of ownership among this group and garnered wide-spread support for the service [38]. Early engagement provided an opportunity to incorporate feedback and concerns early in the development process. For example, concerns were raised around pharmacists having the necessary education to assess and prescribe medicines for minor ailments. It was confirmed that patient assessment for minor ailments is an integral component of the core curricula at all ten faculties of pharmacy across Canada [37]; graduates are equipped with the necessary competencies to support clinical decision making required for minor ailment prescribing. In addition to curricula, continuing education for minor ailment prescribing has been available and continues to be accessed by the profession in preparation for this scope, which has been long-awaited. The mandatory orientation module reinforces the importance of referring patients to other members of the healthcare team for management of issues beyond their competence as well as the need to self-assess continually one's knowledge, skill, and judgement necessary for the provision of safe and quality patient care.

Stakeholder feedback activities were also embedded within the structured process used to prioritize the ailments and informed refinement of the final list. This process also 
included analysis of data from multiple inputs, including the environmental scan, MAAG's modified-Delphi proceedings, and provincial data describing the most prevalent minor ailments resulting in ED visits across Ontario [31]. The latter provided insight on the impact that pharmacist prescribing may have if this type of routine or minor care were moved into the community. A UK study published by Nazar et al. utilized expert consensus through interacting group decision and nominal group process [39]. The group reviewed anonymized patient data and reached consensus on low acuity conditions that could be safely redirected to the community pharmacy through the National Health Services medical helpline. This approach was similar to Ontario's in that an expert group provided advice via consensus by analyzing data and identifying conditions that would be appropriate for pharmacist intervention. In that study, the participants were divided into smaller groups to facilitate parallel discussions to achieve consensus across groups on conditions that required additional discussion, whereas in Ontario, these discussions occurred in the larger group setting. While both considered risk to patient in the prioritization of the conditions, Nazar et al. used the failure mode and effects analysis (FMEA) method to help identify risk, whereas we relied on expert guidance and the conceptual framework to help inform these decisions. There may be value in utilizing a standard risk tool when selecting minor ailments for inclusion in PPMA programs. Further research exploring the impact of such a tool on patient outcomes would be beneficial.

The next step entailed selecting medication categories for pharmacist prescribing in Ontario. Our approach in utilizing the AHFS classification system differs from all other jurisdictions in Canada partly due to our regulatory framework, which does not allow for broad prescriptive authority as is the case in other provinces. The regulations must include either medication names or categories; medication categories were preferred over medication names as categories and offer a more flexible approach than medication lists to ensure pharmacists have access to the most up-to-date medications without requiring a regulatory change. The AHFS system was used to align with how other healthcare professions reference medications in the regulations.

Embedded within our provincial regulations, and consistent with that of most Canadian provinces, is the requirement for pharmacists to notify the PCP of the patient consultation and treatment plan after prescribing; this safeguard differs from other PPMA services globally as it is not always a mandatory element in other countries [6]. However, communication with the PCP was identified by patients during our stakeholder engagement activities as an essential component of the service for continuity of care purposes, cementing its importance in service delivery. Additionally, pharmacies in Ontario are not connected to a provincial electronic medical record, therefore setting out the added expectation for communication with the PCP. The communication channel is not specified, and pharmacists may choose a method established with the PCP.

In Canada, the training requirements to prescribe for minor ailments vary provincially; some jurisdictions (e.g., Nova Scotia) do not require any training, while others mandate a non-clinical orientation to the regulations (e.g., Ontario, New Brunswick), and others have more comprehensive, clinical training requirements (e.g., Saskatchewan) [40]. In Ontario, pharmacists will be required to complete an online orientation to the regulatory requirements and practice expectations. A recent international review examining the evidence regarding training, education, and assessment requirements associated with the delivery of PPMA services by community pharmacists and other pharmacy staff found that $46 \%$ required pharmacists to complete mandatory training, which varied in its nature, time commitment, and delivery (e.g., live, online) [35].

Globally, antimicrobial resistance continues to be a significant public health threat as antibiotics are frequently prescribed for viral illnesses where there is no benefit to patients [41]. Specifically, antibiotic overuse is associated with rising rates of drug-resistant infections, avoidable adverse drug reactions, and increasing healthcare costs [42]. U.S. data estimate that approximately 30 to $50 \%$ of antibiotics prescribed in outpatient settings may be unnecessary $[43,44]$. Enabling pharmacists to prescribe antibiotics to treat common 
infectious diseases offers an opportunity to harness pharmacists as a source of improved rational antibiotic prescribing and patient education, enhancing access to care and ensuring the right patients get antibiotics when necessary. To support pharmacists in this role, the MAAG co-developed practice tools that are based on evidence and antimicrobial stewardship principles to guide assessment and decision-making for patients with UTIs and tick bites.

The evidence of the benefits of pharmacist prescribing of antimicrobials is growing. The RxOUTMAP study found that pharmacist management of uncomplicated UTIs across 39 community pharmacies in New Brunswick was effective and safe, and resulted in high patient satisfaction [29]. Of the 750 patients enrolled, clinical cure was achieved in $88.6 \%$ of those managed by a pharmacist compared to $91.1 \%$ for those managed by a physician $(p>0.99)$, and there were no statistically significant differences in safety outcomes [29]. The time from the patient's decision to seek care until they were seen by a pharmacist or physician was 1.7 vs 2.8 days, respectively ( $p=0.0153$ ). These results are consistent with previous studies on this topic. A smaller Scottish study comparing the care pathways of patients with UTI symptoms found that patients seeking pharmacist management presented earlier than those seeking physician management $(p=0.026)$, and that providing antibiotics for UTIs through strict protocols in community pharmacies improved access to treatment for patients and had the potential to maintain antibiotic stewardship while reducing physician workload [45]. Recognizing this role for pharmacists and considering the healthcare needs, Ontario included UTI in the proposed PPMA program. Other provinces (e.g., Prince Edward Island, Quebec) also recently enabled pharmacists to prescribe for UTIs. These types of evaluative studies helped to inform policy direction in terms of including conditions that involve antimicrobial prescribing.

Implementation science assists with analyzing the most important domains specific to a policy direction to determine how to execute the desired outcome successfully. In our experience, implementation science informed our approach to operationalizing the service by focusing on the key drivers of behavioral change, which includes capability, opportunity, and motivation [46]. During the early engagement activities, stakeholders were surveyed to provide feedback on potential facilitators and barriers to incorporating PPMA into their practice. Coupled with the findings from the environmental scan, the resultant factors were then mapped to the COM-B framework to enhance understanding of the complexity of potential facilitators and barriers, and ultimately identify strategies to assist with implementation. For example, integrating PPMA into practice in a busy environment was noted as a concern by members of the public and pharmacy professionals, which is consistent with the literature [23,47]. Applying this identified barrier to the framework allowed for recognition of the importance of creating an opportunity for these services by making the practice environment more conducive to this type of service.

The COM-B model can be further elaborated by the Theoretical Domains Framework [48], which consists of 14 domains covering the spectrum of behavioral determinants. These domains can be mapped directly onto the COM-B components. In their study, Isenor et al. utilized the Theoretic Domains Framework version 2 to identify the relationship between barriers and facilitators to pharmacist prescribing and self-reported prescribing activity [49]. The three domains that respondents most positively associated with prescribing were knowledge, reinforcement, and intentions. The largest effect on prescribing activity was the skills domain. The study concluded that this understanding could assist the development of policy and program interventions at the pharmacist, pharmacy, and health system levels, to increase the uptake of pharmacist prescribing.

Currently, there seems to be limited evidence about the effectiveness of tailored implementation strategies. In Ontario, the evaluation framework was constructed alongside the implementation strategy in order to evaluate both the outcomes of the PPMA program and the process. The framework will be used to guide evaluators who may be involved in this work. 


\section{Limitations}

The environmental scan was used to triangulate evidence from the peer-reviewed literature, domain experts, and a grey literature search. There are some limitations associated with this type of environmental scan methodology. First, the search was limited to research articles and grey literature on PPMA services across Canada only. This was done to streamline our efforts by focusing our analysis on PPMA features in the Canadian context. Studies were not excluded on the basis of their methodological quality. Second, a portion of information gathering about other PPMA programs in Canada included web searches and databases, which are only as accurate and up-to-date as the information posted on them. Third, a portion of this information was attained through personal communication with individuals who represent various provincial organizations. As such, reproducing the research process may be a challenge. Fourth, despite providing a definition of "minor ailment" at the start of the process, considerable variation was encountered across jurisdictions, ranging from differences in prescribing authority and naming of the services that describe them to diversity in the range of conditions considered to be minor ailments. For example, fungal skin infections are referred to more broadly in some jurisdictions but referred to more specifically by type (e.g., tinea pedis) in other jurisdictions. Another example is UTI. Some jurisdictions define this condition as "uncomplicated urinary tract infection" (e.g., New Brunswick, Ontario), some as "cystitis-acute, uncomplicated" (e.g., Saskatchewan), while others define it as "uncomplicated cystitis" (e.g., Manitoba, Nova Scotia). Furthermore, provinces like Nova Scotia consider prescribing for UTIs under a separate component of the legislation titled "Prescribing for a Diagnosis Supported by a Protocol" rather than under their minor and common ailment regulations [21]. The resulting ambiguity was a limitation of our process as it interfered with our ability to draw accurate comparisons between jurisdictions and apply the generalizations required for a clear summary.

\section{Conclusions}

As pharmacy practice continues to evolve to meet the current and future demands of healthcare, including public health emergencies such as COVID-19, so must the programs (e.g., PPMA services) that enable pharmacists to provide patient care. Pharmacists actively participated in the pandemic response, which highlighted their role and commitment to accessible healthcare. PPMA services offer another opportunity for pharmacists to provide streamlined care pathways for patients.

This paper explored the development of the PPMA service in Ontario from the perspectives of those involved in its design and implementation. Based on our experience, we found that taking an outcomes-focused, systems-based approach by considering the objectives of the program (i.e., improved access to care in the community) and by engaging with stakeholders throughout the healthcare system allowed us to identify key components to the program, identify areas of greatest need, and ensure that quality and safety remain at the core.

Through this work, we are able to offer an academic and policy perspective that may help other policy makers and individuals involved in effective pharmacy service design and implementation. Use of the frameworks (i.e., COM-B, Logic Model) was found to offer valuable insight as explanatory and diagnostic tools in policymaking; these frameworks present an alternative approach to PPMA program design and implementation that considers the potential barriers and enablers while visualizing the objectives and intended outcomes that can be used as a guide for evaluative studies. This also enabled the review of pharmacy curricula to ensure that the components of the PPMA program (e.g., physical and non-physical assessment) are covered in universities prior to licensure and service delivery.

Continual evaluation and review of programs may help inform enhancements to the services necessary to meet the changing demands of healthcare in the years to come. This requires the generation of additional high-quality evidence through the use of adequately 
powered randomized controlled trials or longitudinal observational studies evaluating PPMA programs, which are focused on the use of validated clinical outcome measures [50]. The availability of this type of evidence provides a foundation for informing changes to programs based on emerging healthcare needs. Thus, the PPMA programs should be able to adapt to the dynamic healthcare system and mounting body of evidence, for example, adding conditions to the program in light of new literature proving cost effectiveness and optimal clinical outcomes. Furthermore, additional research may provide robust evaluation data necessary for the comprehensive appreciation of the impact of existing PPMA programs both nationally and internationally.

Author Contributions: Conceptualization-N.N. and A.S.; methodology, N.N. and A.S.; formal analysis, N.N. and A.S.; investigation, N.N. and A.S.; resources, N.N. and A.S.; data curation, N.N. and A.S.; writing-original draft preparation, N.N. and A.S.; writing—review and editing, N.N. and A.S.; visualization, N.N. and A.S.; supervision, A.S.; project administration, N.N. Both authors have read and agreed to the published version of the manuscript.

Funding: This research received no external funding.

Institutional Review Board Statement: Not applicable.

Data Availability Statement: Data related to this article can be found at https:/ /www.ocpinfo.com/ wp-content/uploads/2020/12/Minor-Ailments-Advisory-Group-MAAG-Summary-of-Recommen dations-Pharmacist-Prescribing.pdf.

Acknowledgments: The authors would like to acknowledge and thank the Ontario College of Pharmacists for supporting this publication and the members of the Minor Ailments Advisory Group (MAAG) for their guidance in developing the draft regulations with a view of improving health outcomes and health system quality while ensuring patient safety and in the context of broader engagement.

Conflicts of Interest: The authors declare no conflict of interest.

\section{Appendix A}

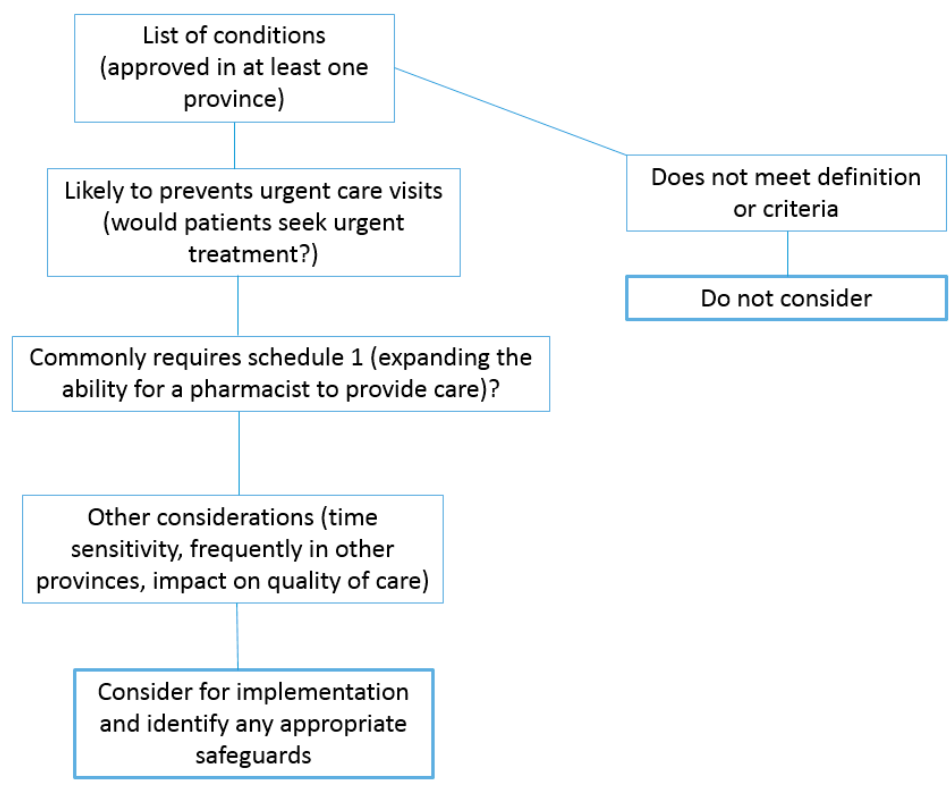

Figure A1. Conceptual framework. 


\section{References}

1. National Statistics. National Association of Pharmacy Regulatory Authorities (NAPRA). Available online: https://napra.ca/ national-statistics (accessed on 1 February 2021).

2. Taylor, J.G.; Joubert, R. Pharmacist-led minor ailment programs: A Canadian perspective. Int. J. Gen. Med. 2016, 9, 291-302. [CrossRef] [PubMed]

3. Fielding, S.; Porteous, T.; Ferguson, J.; Maskrey, V.; Blyth, A.; Paudyal, V.; Barton, G.; Holland, R.; Bond, C.M.; Watson, M.C. Estimating the burden of minor ailment consultations in general practices and emergency departments through retrospective review of routine data in North East Scotland. Fam. Pract. 2015, 32, 165-172. [CrossRef]

4. Paudyal, V.; Watson, M.C.; Sach, T.; Porteous, T.; Bond, C.M.; Wright, D.J.; Cleland, J.; Barton, G.; Holland, R. Are pharmacy-based minor ailment schemes a substitute for other service providers? A systematic review. Br. J. Gen. Pract 2013, 63, e472-81. [CrossRef] [PubMed]

5. Paudyal, V.; Hansford, D.; Cunningham, S.; Stewart, D. Pharmacy assisted patient self care of minor ailments: A chronological review of UK health policy documents and key events 1997-2010. Health Policy 2011, 101, 253-259. [CrossRef]

6. Aly, M.; Garcia-Cardenas, V.; Williams, K.; Benrimoj, S.I. A review of international pharmacy-based minor ailment services and proposed service design model. Res. Social Adm. Pharm. 2018, 14, 989-998. [CrossRef]

7. Watson, M.C.; Holland, R.; Ferguson, J.; Porteous, T.; Sach, T.; Cleland, J. Community Pharmacy Management of Minor Illness (the MINA Study). In Final Report to Pharmacy Research UK; Pharmacy Research UK: London, UK, 2014. Available online: https:/ / pharmacyresearchuk.org/wp-content/uploads/2014/01/MINA-Study-Final-Report.pdf (accessed on 3 February 2021).

8. Watson, M.C.; Ferguson, J.; Barton, G.R.; Maskrey, V.; Blyth, A.; Paudyal, V.; Bond, M.C.; Holland, R.; Porteous, T.; Sach, T.H.; et al. A cohort study of influences, health outcomes and costs of patients' health-seeking behaviour for minor ailments from primary and emergency care settings. BMJ Open 2015, 5, e006261. [CrossRef] [PubMed]

9. Scottish Government Health and Social Care Directorates. National Health Service (Scotland) Act 1978: Health Board Additional Pharmaceutical Services (Minor Ailment Service) Amendment Directions 2020. Available online: https://www.sehd.scot.nhs.uk/ pca/PCA2020(P)06.pdf (accessed on 1 February 2021).

10. Information Services Division. "Prescribing \& Medicines: Minor Ailments Service (MAS)." NHS National Services Scotland. Available online: https:/ / www.isdscotland.org/Health-Topics/Prescribing-and-Medicines/Publications/2017-09-26/2017-09 -26-Prescribing-MinorAilmentsService-Report.pdf (accessed on 2 February 2021).

11. Dineen-Griffin, S.; Garcia-Cardenas, V.; Rogers, K.; Williams, K.; Benrimoj, S.I. Evaluation of a Collaborative Protocolized Approach by Community Pharmacists and General Medical Practitioners for an Australian Minor Ailments Scheme: Protocol for a Cluster Randomized Controlled Trial (in eng). JMIR Res. Protoc. 2019, 8, e13973. [CrossRef]

12. Sewak, N.; Cairns, J. A modelling analysis of the cost of a national minor ailments scheme in community pharmacies in England. Int. J. Pharm. Pract. 2011, 19, 50.

13. Business Services Organisation. "Minor Ailments Scheme". Health and Social Care Board. Available online: http://www. hscbusiness.hscni.net/services/2055.htm (accessed on 1 February 2021).

14. Common Ailments Service. Cwm Taf Morgannwg University Health Board. Available online: https://cwmtafmorgannwg. wales/services/pharmacy-medicines-management/common-ailments-service/ (accessed on 1 February 2021).

15. Welsh Government. Community Pharmacy Common Ailment Service-Service Specification. Available online: http://www. wales.nhs.uk/sites3/Documents /498/CAS\%20Service\%20Specification.pdf (accessed on 3 February 2021).

16. Dineen-Griffin, S.; Vargas, C.; Williams, K.A.; Benrimoj, S.I.; Garcia-Cardenas, V. Cost utility of a pharmacist-led minor ailment service compared with usual pharmacist care. Cost Eff. Resour. Alloc. 2020, 18, 24. [CrossRef]

17. Ontario College of Pharmacists. Expanded Scope of Practice. Available online: https://www.ocpinfo.com/about/key-initiatives/ expanded-scope-of-practice/ (accessed on 1 September 2020).

18. Ontario Ministry of Health and Long-Term Care. First Interim Report from the Premier's Council on Improving Healthcare and Ending Hallway Medicine. Available online: http:/ /www.health.gov.on.ca/en/public/publications/premiers_council/report. aspx (accessed on 1 February 2021).

19. Minor Ailments Advisory Group (MAAG). Summary of Recommendations: Pharmacist Prescribing for Minor Ailments. Ontario College of Pharmacists. Available online: https:/www.ocpinfo.com/wp-content/uploads/2020/12/Minor-Ailments-AdvisoryGroup-MAAG-Summary-of-Recommendations-Pharmacist-Prescribing.pdf (accessed on 1 February 2021).

20. Pharmacy Association of Nova Scotia. Assessing and Prescribing for Minor Ailments. Available online: https://pans.ns.ca/ public/pharmacy-services/assessing-prescribing-minor-ailments (accessed on 1 February 2021).

21. Nova Scotia College of Pharmacists. Standards of Practice: Prescribing Drugs. Available online: https://www.nspharmacists.ca/ wp-content/uploads/2016/05/SOP_PrescribingDrugs.pdf (accessed on 2 February 2021).

22. Cramm, D.; Kent, S. Authorization to Prescribe Regulations 2015. Government of Newfoundland and Labrador. Available online: https:/ / www.assembly.nl.ca/legislation/sr/annualregs/2015/nr150073.htm (accessed on 2 February 2021).

23. Shearer, B.; Ng, S.; Dunford, D.; Kuo, I.F. Training Needs of Manitoba Pharmacists to Increase Application of Assessment and Prescribing for Minor Ailments into Practice: A Qualitative and Quantitative Survey. Pharmacy 2018, 6, 82. [CrossRef]

24. Bishop, A.C.; Boyle, T.A.; Morrison, B.; Barker, J.R.; Zwicker, B.; Mahaffey, T.; Murphy, A. Public perceptions of pharmacist expanded scope of practice services in Nova Scotia. Can. Pharm. J. 2015, 148, 274-283. [CrossRef] [PubMed] 
25. Feeley, D. The Triple Aim or the Quadruple Aim? Four Points to Help Set Your Strategy. Institute for Healthcare Improvement. Available online: http:/ / www.ihi.org/communities/blogs/the-triple-aim-or-the-quadruple-aim-four-points-to-help-set-yourstrategy (accessed on 3 February 2021).

26. Ontario Ministry of Health. Chapter 2: The vision for health care in Ontario. Available online: https://www.ontario.ca/ document/healthy-ontario-building-sustainable-health-care-system/chapter-2-vision-health-care-ontario (accessed on 1 February 2021).

27. Mansell, K.; Bootsman, N.; Kuntz, A.; Taylor, J. Evaluating pharmacist prescribing for minor ailments. Int. J. Pharm. Pract. 2015, 23, 95-101. [CrossRef] [PubMed]

28. Research Power Inc. Evaluation of the Provision of Minor Ailment Services in the Pharmacy Setting Pilot Study: Final Report. Pharmacy Association of Nova Scotia (PANS). Available online: https://pans.ns.ca/wp-content/uploads/2013/11/2013-10-17 -PANS-report_FINAL.pdf (accessed on 2 February 2021).

29. Beahm, N.P.; Nicolle, L.E.; Bursey, A.; Smyth, D.J.; Tsuyuki, R.T. The assessment and management of urinary tract infections in adults: Guidelines for pharmacists. Can. Pharm. J. 2017, 150, 298-305. [CrossRef] [PubMed]

30. Taylor, J.G.; Mansell, K. Patient Feedback on Pharmacist Prescribing for Minor Ailments in a Canadian Province. Inov. Pharm. 2017, 8, 1. [CrossRef]

31. Alsabbagh, M.W.; Houle, S.K.D. The proportion, conditions, and predictors of emergency department visits that can be potentially managed by pharmacists with expanded scope of practice. Res. Social Adm. Pharm. 2019, 15, 1289-1297. [CrossRef]

32. Citizen Advisory Group (CAG). Report: Citizen Advisory Group Meeting. Saturday 8 February 2020. Available online: https: / / citizenadvisorygroup.files.wordpress.com/2020/04/ citizen-advisory-group-8-feb-2020-final-report.pdf (accessed on 3 February 2021).

33. Minor Ailments. Ontario College of Pharmacists. Available online: https://www.ocpinfo.com/practice-education/expandedscope-of-practice/minor-ailments / ?hilite $=\% 27$ minor $\% 27 \% 2 \mathrm{C} \% 27$ ailments $\% 27$ (accessed on 2 February 2021).

34. Public Health Ontario. Ontario Lyme Disease Map 2020 Estimated Risk Areas. Available online: https: / /www.publichealthontario. $\mathrm{ca} /$ - /media/documents/1/2020/lyme-disease-risk-area-map-2020.pdf (accessed on 1 March 2021).

35. Aly, M.; Schneider, C.R.; Sukkar, M.B.; Lucas, C. Educational needs of community pharmacy staff in minor ailment service delivery: A systematic scoping review. Curr. Pharm. Teach. Learn. 2020, 12, 1269-1287. [CrossRef]

36. Nazar, H.; Nazar, Z. Community pharmacy minor ailment services in England: Pharmacy stakeholder perspectives on the factors affecting sustainability. Res. Soc. Adm. Pharm. 2019, 15, 292-302. [CrossRef] [PubMed]

37. Nakhla, N.; Black, E.; Abdul-Fattah, H.; Taylor, J. Self-care education across Canadian pharmacy schools: Curriculum survey findings. Can. Pharm. J. 2021, 154, 52-60. [CrossRef]

38. Mantzourani, E.; Richards, T.G.; Hughes, M.L. New roles in pharmacy-learning from the All Wales Common Ailments Scheme. Int. J. Pharm. Pract. 2016, 24, 298-301. [CrossRef]

39. Nazar, H.; Nazar, Z.; Yeung, A.; Maguire, M.; Connelly, A.; Slight, S.P. Consensus methodology to determine minor ailments appropriate to be directed for management within community pharmacy (in eng). Res. Social. Adm. Pharm. 2018, 14, 1027-1042. [CrossRef]

40. Habicht, D.; Ng, S.; Dunford, D.; Shearer, B.; Kuo, I.F. Incorporating assessment and prescribing for ambulatory ailments skills into practice: An environmental scan of continuing education for pharmacist prescribing in Canada. Can. Pharm. J. 2017, 150, 316-325. [CrossRef]

41. Public Health Agency of Canada. Canadian Antimicrobial Resistance Surveillance System 2017 Report. Government of Canada. Available online: https://www.canada.ca/en/public-health/services/publications/drugs-health-products / canadianantimicrobial-resistance-surveillance-system-2017-report-executive-summary.html (accessed on 21 February 2021).

42. Chief Public Health Officer of Canada's 2019 Spotlight Report. Handle With Care: Preserving Antibiotics Now and Into the Future. Public Health Agency of Canada. Available online: https://www.canada.ca/content/dam/phac-aspc/documents/ corporate/publications/chief-public-health-officer-reports-state-public-health-canada/preserving-antibiotics/Final_CPHO_ Report_EN_June6_2019.pdf (accessed on 21 February 2021).

43. Fleming-Dutra, K.E.; Hersh, A.L.; Shapiro, D.J.; Bartoces, M.; Enns, E.A.; File, T.M., Jr.; Finkelstein, J.A.; Gerber, J.S.; Hyun, D.Y.; Linder, J.A.; et al. Prevalence of Inappropriate Antibiotic Prescriptions Among US Ambulatory Care Visits, 2010-2011. JAMA 2016, 315, 1864-1873. [CrossRef] [PubMed]

44. Chua, K.P.; Fischer, M.A.; Linder, J.A. Appropriateness of outpatient antibiotic prescribing among privately insured US patients: ICD-10-CM based cross sectional study. BMJ 2019, 364, 5092. [CrossRef]

45. Booth, J.L.; Mullen, A.B.; Thomson, D.A.M.; Johnston, C.; Galbraith, S.J.; Bryson, S.M.; McGovern, E.M. Antibiotic treatment of urinary tract infection by community pharmacists: A cross-sectional study. Br. J. Gen. Pract. 2013, 63, 244-249. [CrossRef]

46. Michie, S.; Atkins, L.; West, R. The Behaviour Change Wheel: A Guide to Designing Interventions; Silverback Publishing: London, UK, 2014.

47. Tsao, N.W.; Lynd, L.D.; Gastonguay, L.; Li, K.; Nakagawa, B.; Marra, C.A. Factors associated with pharmacists' perceptions of their working conditions and safety and effectiveness of patient care. Can. Pharm. J. 2016, 149, 18-27. [CrossRef] [PubMed]

48. Cane, J.; O'Connor, D.; Michie, S. Validation of the theoretical domains framework for use in behaviour change and implementation research. Implement. Sci. 2012, 7, 37. [CrossRef] [PubMed] 
49. Isenor, J.E.; Minardf, L.V.; Stewart, S.A.; Curran, J.A.; Deal, H.; Rodrigues, G. Identification of the relationship between barriers and facilitators of pharmacist prescribing and self-reported prescribing activity using the theoretical domains framework. Res. Social. Adm. Pharm. 2018, 14, 784-791. [CrossRef]

50. Paudyal, V.; Cunningham, S.; Gibson Smith, K.; MacLure, K.; Ryan, C.; Cordina, M. Methodological considerations in clinical outcomes assessment of pharmacy-based minor ailments management: A systematic review (in eng). PLoS ONE 2018, 13, e0205087. [CrossRef] 